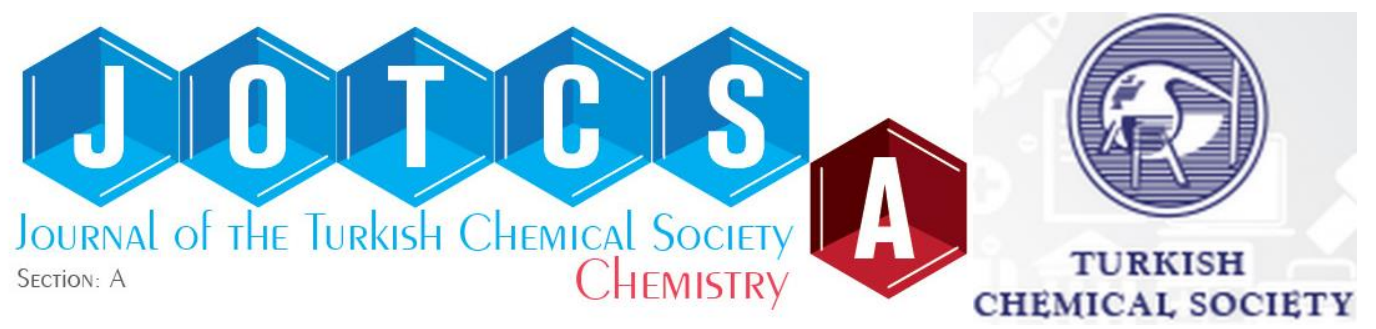

\title{
EFFECT Of IONIC LIQUID CONTENT on the MONOLITHIC STRUCTURE of AMINE-MEDIATED SILICA AEROGEL via AMBIENT PRESSURE DRYING
}

\author{
Nilay GİZLi*, Selay SERT ÇOK, Fatoş KOÇ
}

Ege University, Faculty of Engineering, Department of Chemical Engineering, 35100, İzmir, Turkey.

\begin{abstract}
In this study, amine-mediated silica aerogels dried under the ambient conditions in monolithic form were prepared by following sol-gel method. 3-aminopropyltriethoxysilane (APTES) was involved in the synthesis as a silica co-precursor. Imidazolium-based short-chain ionic liquids (ILs) were incorporated into the silica gel structure to control the gel shrinkage within the pores, as well as to eliminate the capillary effect during the solvent evaporation. A production procedure was developed to explore the synergistic effect of ionic liquids and amine-functionalized silica precursor on the textural and chemical properties of the final silica gels. Surface modifications of the samples were performed by using 3Methacryloxypropyltrimethoxysilane (MEMO) to ensure hydrophobic characteristics. To reveal the chemical and morphological characteristics of the resultant material, various analyses were conducted. SEM and FTIR analyses were performed to investigate the morphological and chemical structure, whereas TGA analysis was carried out to determine the thermal stability of the silica gels. As a result, the ionic liquid embedded sample was obtained in a monolithic structure with a low density $\left(0.45 \mathrm{~g} / \mathrm{cm}^{3}\right)$ and had a good thermal stability (up to $381{ }^{\circ} \mathrm{C}$ ). Contact angle measurement also demonstrated that the desired monolithic sample has a hydrophobic characteristic with a water contact angle value of $113^{\circ}$.
\end{abstract}

Keywords: Ionic liquid, silica aerogel, APTES, ambient pressure drying, monolithic structure.

Submitted: February 5, 2018. Accepted: March 26, 2018.

Cite this: Gizli N, Sert Çok S, Koç F. EFFECT of IONIC LIQUID CONTENT on the MONOLITHIC STRUCTURE of AMINE-MEDIATED SILICA AEROGEL via AMBIENT PRESSURE DRYING. JOTCSA. $2018 ; 5(2): 663-78$.

DOI: http://dx.doi.org/10.18596/jotcsa.390372.

*Corresponding author. E-mail: nilay.gizli@ege.edu.tr 
Gizli N, Sert Çok S, Koç F. JOTCSA. 2018, 5(2): 663-678.

\section{INTRODUCTION}

Silica aerogels are exceptional porous materials with splendid physical properties such as very low density $\left(0.003-0.5 \mathrm{~g} / \mathrm{cm}^{3}\right)$, notably high surface area $\left(500-1200 \mathrm{~m}^{2} / \mathrm{g}\right)$, and high porosity (80\%-99.8\%) (1-4). Due to their outstanding properties aerogels are become a promising candidate in a wide range of applications such as thermal insulation in the construction sector (5), catalysis, chemical sensors (6), drug delivery systems (7), acoustic impedance (8), and space applications (9). They can be produced in various forms (i.e. powders, granular, or monolithic) by changing the synthesis conditions. Silica aerogels in monolithic form have wider applications as it allows controlling final shape of the product for any particular applications. Hence, production of silica aerogel as monolithic blocks can promote the commercialization of these materials.

The silica aerogels are mainly synthesized with the traditional sol-gel method. Typical sol-gel chemistry consists of consecutive hydrolysis and condensation reactions of a silicon alkoxide group in a liquid solvent with proper acid and/or base catalysis to form a colloidal solution called "sol". This process is followed by gelation of the sol, aging of the gel, and then drying (10). Several parameters affect the evolution of the microstructural pattern of the aerogel network, such as the type and amount of precursors, catalyst concentration, type of solvent, and reaction temperature and $\mathrm{pH}$ of sol (7). Aging is also an important step that allows continuation of condensation reactions of some monomers and unreacted $\mathrm{Si}-\mathrm{OR}$ and $\mathrm{Si}-\mathrm{OH}$ groups. Hence, it strengthens the structural gel network. However, drying is the most crucial step to satisfy the desired properties of the final product. The main challenge of drying is to perform it without allowing the collapse of a 3D network of the gel. During drying, development of capillary tension in pore walls causes a shrinkage of the gel body and crack the gel (7). This problem generally can be overcome by $\mathrm{CO}_{2}$ supercritical drying. Although supercritical drying technique is used to avoid the destruction of the porous structure, it is unable to proceed beyond the small-scale batch type applications as it is both an expensive and risky process due to its high-pressure requirement. Therefore, most of the recent studies mainly focus on producing silica aerogels by ambient pressure drying (5-9). Generally, during the production of silica aerogels under ambient conditions, gel shrinkage arises from organic solvent evaporation during aging period and pore destruction and the collapse of the network occurs during the drying period. So, the resulting product often exhibits a low degree of monolithicity (16). Hence, several modifications can be applied to overcome these circumstances such as:

1. Using co-precursor to accelerate the condensation reaction rate during aging to be able to develop a stable network.

2. Adding target specific agents to control the gel shrinkage within the pores by satisfying a "spring back effect" on the pore walls. 
Gizli N, Sert Çok S, Koç F. JOTCSA. 2018, 5(2): 663-678.

RESEARCH ARTICLE

3. Exchanging solvents to eliminate the capillary effect by minimizing surface tension on the liquid and vapor interface.

Gel shrinkage, which is the main obstacle obtaining crack-free monolithic silica aerogel, usually arises from organic solvent evaporation during the aging period. This situation can be prevented by replacing organic solvent with some other solvent that has a lower vapor pressure. Ionic liquids are organic salts with an extremely low vapor pressure over a large temperature range $\left(-96\right.$ to $\left.400^{\circ} \mathrm{C}\right)$, therefore they do not evaporate during long aging periods (10). For that reason, using ionic liquids in the sol-gel process may yield a well-ordered pore structure and a stable gel network. They composed of a large organic cation group such as ammonium, phosphonium, pyridinium, imidazolium, etc. and inorganic/organic anion group. Due to their wide range of cation-anion combinations, ionic liquids can be produced with tunable properties and applied in diverse applications (17). Zhang et al. used various room temperature ionic liquids as templates in the synthesis of mesoporous silica gels and reported that increasing the alkyl chain length on the imidazolium cation can increase the pore size of silica gel and lead to slightly enlarged pores (18). Ivanova et al. produced silica ionogels with a one-step sol-gel method dried with supercritical $\mathrm{CO}_{2}$ by using imidazolium-based ionic liquids with different anionic groups. They stated that each ionic liquid behaved as a co-catalyst during the sol-gel process and reduced the required gelation time (17). Same authors in another study denoted that depending on the functional groups of ILs incorporated in aerogel structure can lead to tailor-made porous materials (19). Karout and Pierre investigated the effect of ionic liquids on silica aerogel produced by partial evaporative drying followed by supercritical drying and stated that shrinkage of xerogels depended largely on the IL volume present in the gel before drying (20). They also investigated the effect of four kinds of ionic liquids on the silica gelation time and indicated that ionic liquids combine a double effect of condensation catalysts of silica and moderator of the condensation rate (21).

Dorcheh and Abbasi reported that acid-catalyzed hydrolysis and condensation lead to weakly branch and microporous structure, whereas basic conditions or two-step acid-base processes increase crosslinking, leading to decrease microporosity and a broader distribution of larger pores in silica gels. However, silica aerogels with larger pores usually have a lower specific surface area (4). In another study, Dourbash et al. explained that although ammonium hydroxide is the most used basic catalyst in the preparation of alcogels, it prolongs the gelation time and usually results in a dense structure. Instead of ammonium hydroxide, they recommended the fluoride-based catalysis to reduce the gelation time (22). On the other hand, Yang et al. prepared a silica aerogel using a two-step process in which a silica coprecursor containing an amine group 3-aminopropyltriethoxysilane (APTES) was involved to the sol. They reported that amine-rich APTES behaved like a basic catalyst and eliminated the additional requirement for a base catalyst during condensation reactions (15). 
In this study, the main purpose is to prepare monolithic silica aerogels mediated with APTES under ambient conditions. It is also aimed to explore the synergistic spring back effect of adding ionic liquid into the sol-gel process on the morphological and chemical structure of the final product. Additionally, the reciprocal influence of ionic liquid and APTES on each other through the synthesis is evaluated with various characterizations for the first time to the best of our knowledge.

\section{EXPERIMENTAL PROCEDURE}

\section{Materials}

Tetraethylorthosilicate (TEOS) as precursor and 3-aminopropyltriethoxy-silane (APTES) as coprecursor were acquired from Sigma Aldrich. 1-Ethyl-3-methylimidazolium bis(trifluoromethanesulfonyl)imide (EMIMTF2N) was utilized as the short-chain imidazoliumbased ionic liquid, and 3-methacryloxypropyltrimethoxysilane (MEMO) as surface modification agent were purchased from Sigma Aldrich. Ethanol (EtOH) and n-hexane were used as solvents and hydrochloric acid $(\mathrm{HCl})$ was used as the acid catalyst.

\section{Preparation of silica aerogels}

The silica aerogels were prepared by following one-step sol-gel processes with or without ionic liquid. For comparion purposes, silica aerogels were prepared in the absence (SG) or presence (SG-IL) of ionic liquid. During the preparation of the first sample SG-IL, TEOS was hydrolyzed by using $0.01 \mathrm{M} \mathrm{HCl}$ for $90 \mathrm{~min}$ by stirring at $25^{\circ} \mathrm{C}$. Hydrolysis reaction can take place as follows:

$$
\begin{gathered}
\text { TEOS + Water } \leftrightarrow \text { Silanol + Ethanol } \\
\left(\mathrm{C}_{2} \mathrm{H}_{5} \mathrm{O}\right)_{4}-\mathrm{Si}+\mathrm{H}_{2} \mathrm{O} \leftrightarrow \mathrm{Si}-(\mathrm{OH})_{4}+\mathrm{C}_{2} \mathrm{H}_{5} \mathrm{OH}
\end{gathered}
$$

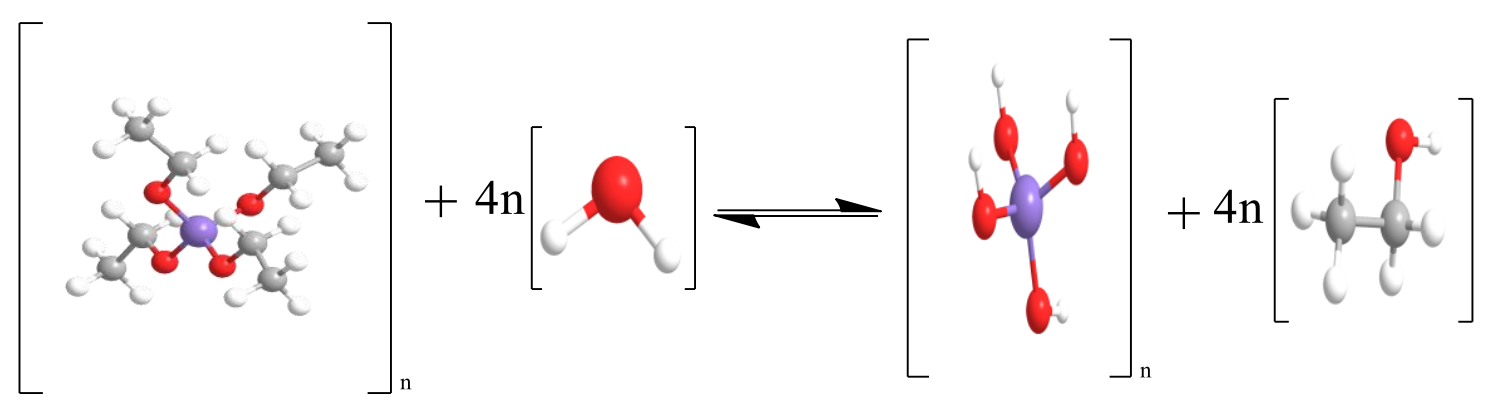

EtOH was used as a solvent whereas imidazolium-based ionic liquid was involved in the sol to serve as target-specific agent. After stirring for 90 minutes, the sol was placed in an ultrasonic media and APTES was added to the sol to start condensation reaction. It can be demonstrated as given below: 


$$
\left(\mathrm{C}_{2} \mathrm{H}_{5} \mathrm{O}\right)_{4}-\mathrm{Si}+\mathrm{Si}-\left(\mathrm{C}_{2} \mathrm{H}_{5} \mathrm{O}\right)_{4} \rightarrow \mathrm{R}_{1}-(-\mathrm{Si}-\mathrm{O}-\mathrm{Si}-)_{n}-\mathrm{R}_{2}+\mathrm{H}_{2} \mathrm{O}
$$
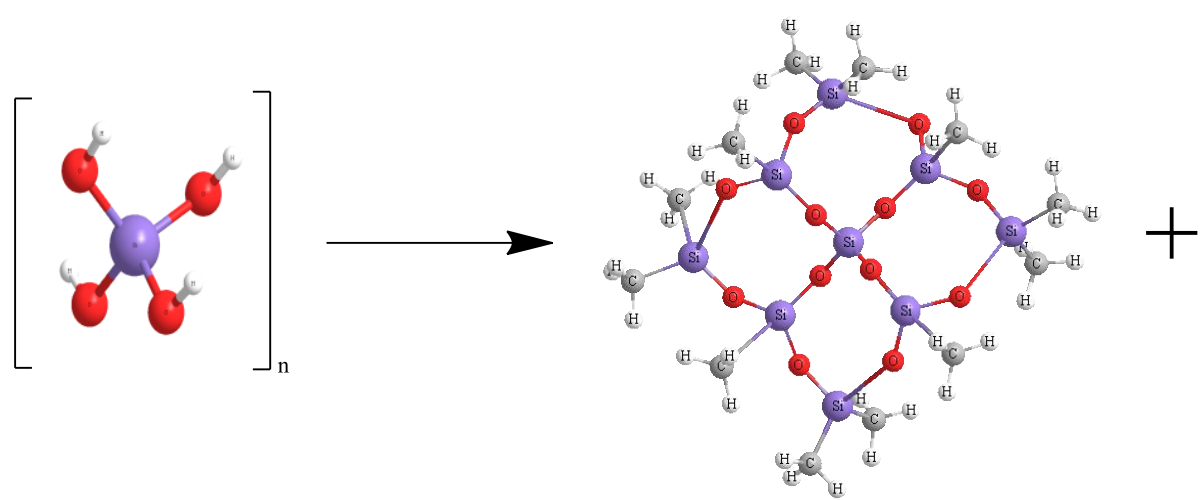

The sol component consists of TEOS: APTES: IL: EtOH: $\mathrm{HCl}$ with the molar ratios of $1: 0.47: 0.14: 6.3: 7.4 \times 10^{-5}$. After complete gelation, the sample was allowed to age for $24 \mathrm{~h}$ in a polypropylene cylindrical mold to further continuation of the condensation reaction. Subsequently, the solvent exchange was carried out by treating the wet gels with fresh $\mathrm{n}$ hexane for $12 \mathrm{~h}$ to ensure complete removal of the impurities within the gel. Then, surface modification was conducted by immersing the sample in silylating agent MEMO diluted with $\mathrm{n}$ hexane at a volumetric percent of $50 \%$ at room temperature. Surface modification reaction can be shown as follows;

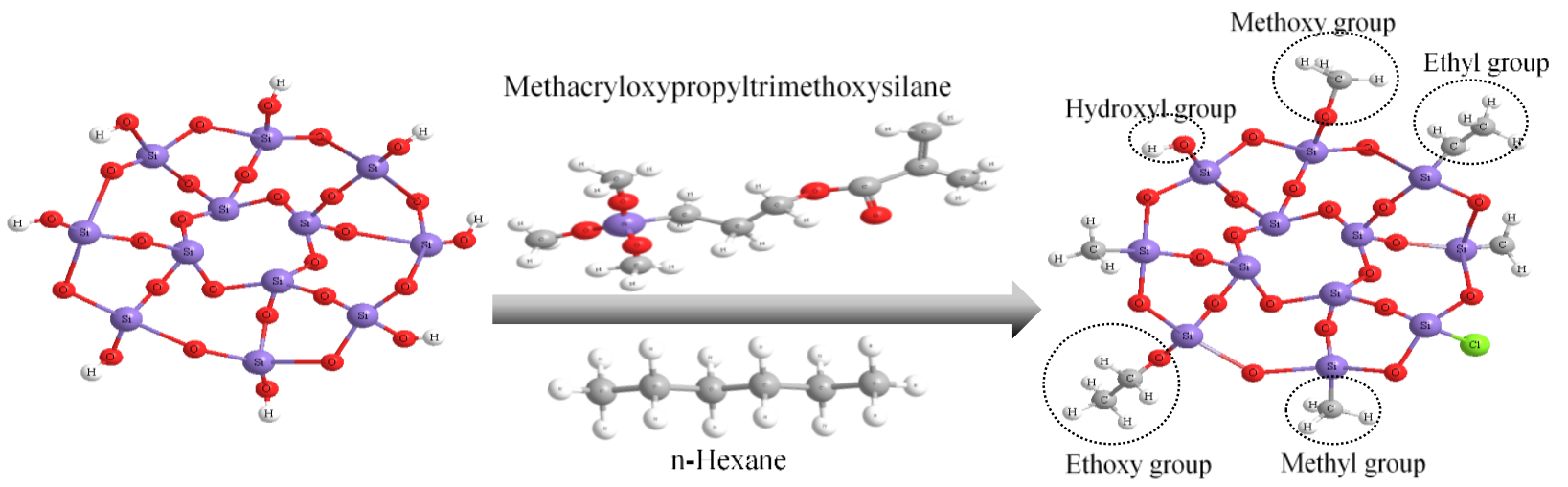

Finally, the sample SG-IL was dried in atmospheric media for two days. During the preparation of the second sample SG, the same procedure was followed with one difference that, unlike SG-IL, sample SG did not include ionic liquid in the sol. The sol components molar ratio for SG

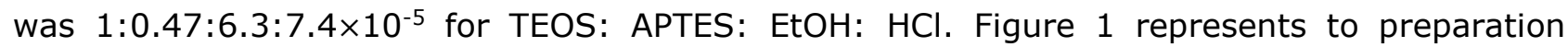
steps of the samples by following sol-gel method. 

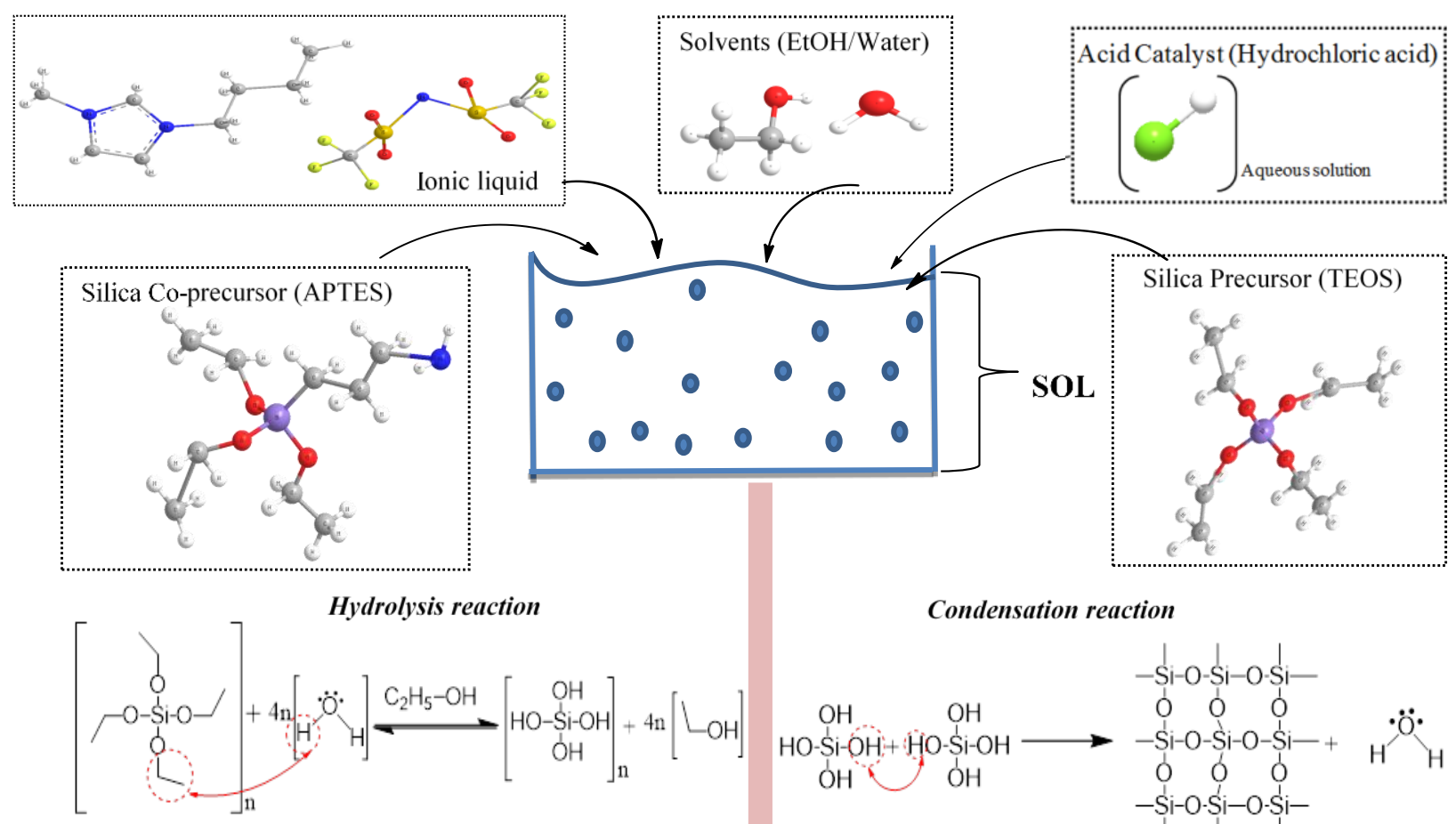

\section{Condensation reaction}
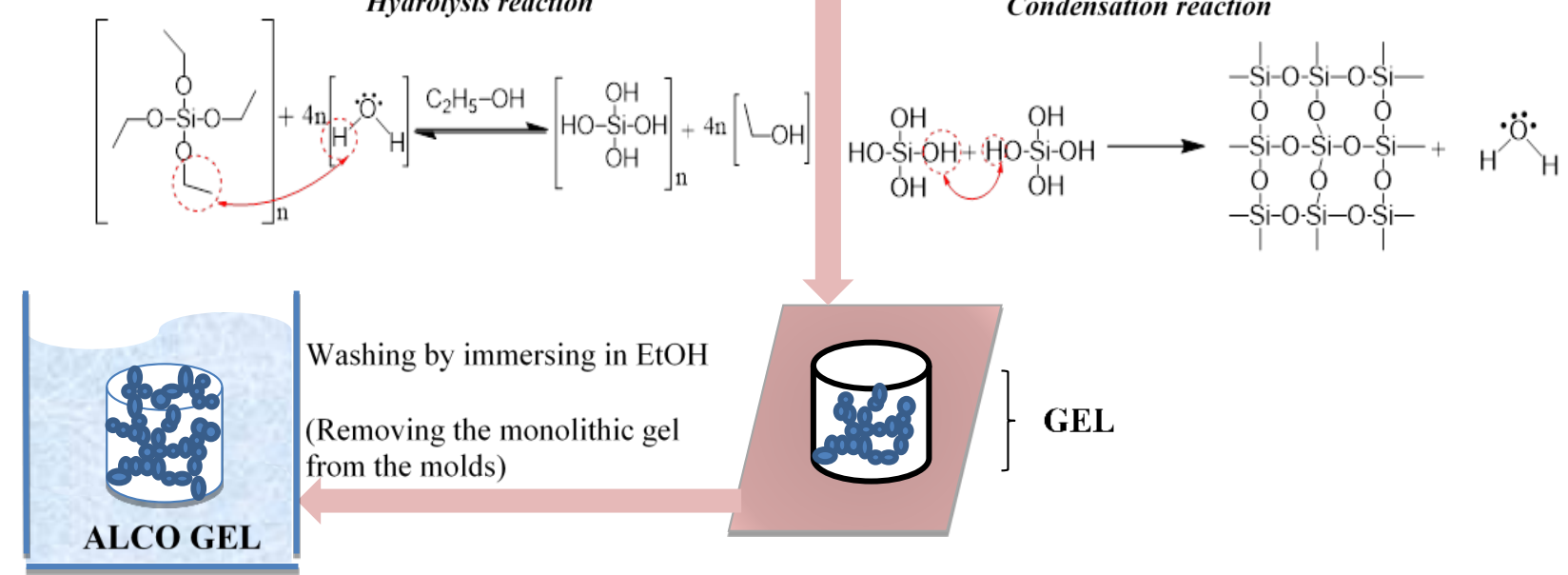

GEL

Surface

Modification

(Contacting

with MEMO

+ n-Hexane

solution)
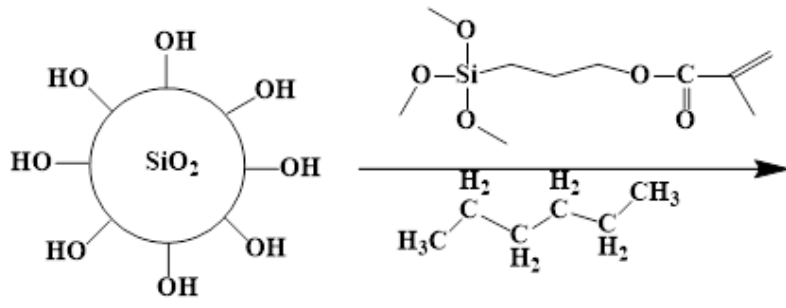

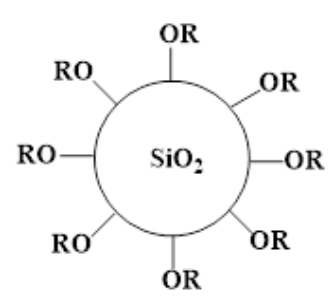

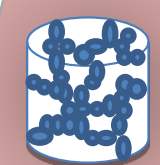

WET GEL

Drying at ambient conditions (removing of solvent from the pores)
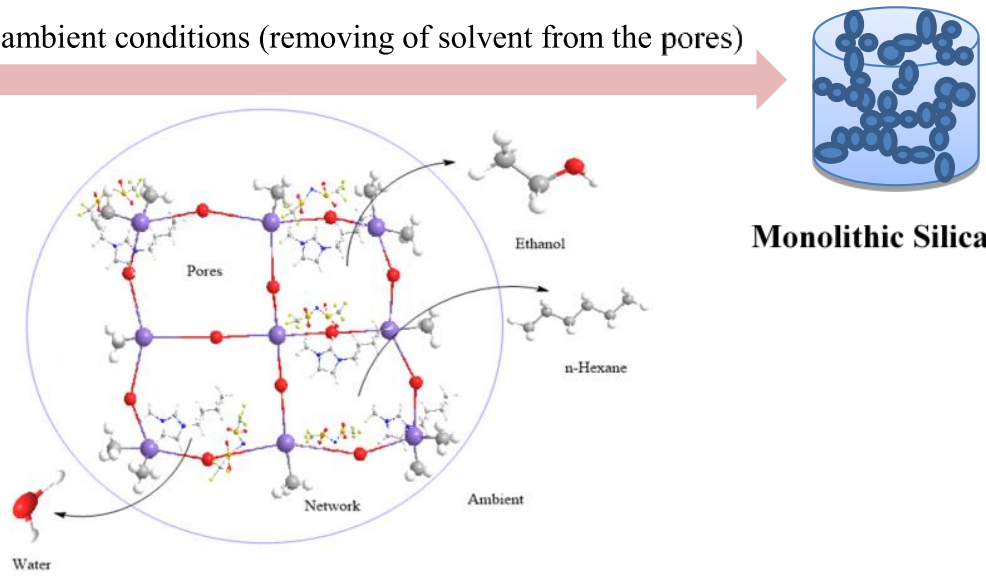

Monolithic Silica Aerogels

Figure 1 Sol-gel preparation steps. 


\section{Characterization}

Bulk densities of dried gels were determined from mass to volume ratio (Eq. 1). The percentages of volume shrinkage and porosity of the samples were estimated according to equations below (Eq. 2, Eq. 3):

$$
\begin{aligned}
& \rho_{b}\left(\mathrm{~g} / \mathrm{cm}^{3}\right)=m_{\text {bulk }} / V_{\text {bulk }} \\
& \text { \%Porosity }=\left(1-\rho_{b} / \rho_{s}\right) \times 100 \\
& \text { \%Volume shrinkage }=\left(1-V_{a} / V_{g}\right) \times 100
\end{aligned}
$$

where $\rho_{b}$ is the bulk density of synthesized silica aerogel and $\rho_{s}$ is the density of solid skeleton. With respect to former studies $\rho_{s}$ can be assumed as $(2.2 \mathrm{~g}) / \mathrm{cm}^{3}(1) . V_{a}$ and $V_{g}$ in the Eq. 3 denote the volumes of the samples after drying and before the solvent exchange, respectively $(4,23)$.

Fourier Transform Infrared Spectroscopy (FTIR) (PERKIN ELMER Spectrum 100, USA) was used between the wavenumber range of $500-4000 \mathrm{~cm}^{-1}$ to identify the chemical compositions of the samples.

The morphology of the samples was observed by using scanning electron microscope (SEM) (PHILIPS, XL 30S FEG) with a magnification rate of 5000 .

To investigate the thermal stability of the samples, thermo-gravimetric analysis (TGA) was performed using a TA Instruments SDT Q600 operating at a heating rate of $5{ }^{\circ} \mathrm{C} / \mathrm{min}$ from room temperature to $600^{\circ} \mathrm{C}$ under a nitrogen atmosphere.

Hydrophobicity of the aerogels was examined by measuring the contact angle $(\theta)$ of the water droplet with the sample surface.

$$
\theta=2 \tan ^{-1}\left(\frac{2 h}{w}\right)
$$

where $\mathrm{h}$ is the height and $\mathrm{w}$ is the width of the water droplet touching the aerogel surface. Travelling micropores were used for measuring $h$ and $w$ (24). Figure 2 demonstrates that small values of contact angle $\left(<90^{\circ}\right)$ correspond to high wettability whereas large contact angles represent hydrophobicity. 


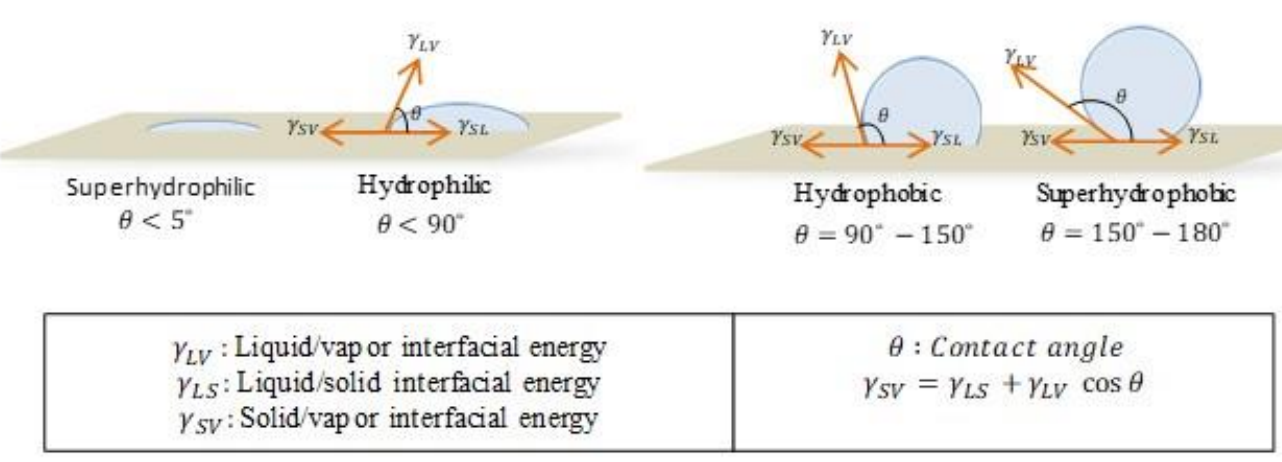

Figure 2: Water drop - surface contact angle.

\section{RESULTS and DISCUSSION}

Up to now, many studies have been directed their attention toward the enhancement of aerogel production under ambient conditions for further commercialization of these materials. One of the key issues about ambient pressure drying in aerogel production is to conserve monolithic structure during drying. Hence, removing of entrapped solvents from the wet gel while avoiding shrinkage and maintaining the porous structure become today's prior challenge. Imidazolium-based ionic liquid incorporated into silica aerogel synthesis in this study with the intention of promoting spring-back effect against deterioration of porous structure during ambient drying. On the other hand, an amine-containing silica co-precursor APTES was involved in the preparation of aerogels for the purpose of reducing required time for synthesis. To explore the interaction between ionic liquid and APTES, two different samples were prepared using same components and same procedure with or without ionic liquid and various physical and chemical characterizations were conducted as follows.

\section{Physical Properties}

To compute the density values of the samples with standart errors by using Eq.1, the weight and volume of the samples was measured three times. Calculated density, porosity and percentage of volumetric shrinkage of the SG-IL and SG were tabulated in Table 1 with standart deviations. The sample SG-IL had small volume shrinkage and lower density ( $\rho s G-$ $\mathrm{IL}=0.45 \mathrm{~g} / \mathrm{cm}^{3}$ ) whether the sample SG could not resist the capillary effect.

Table 1. Observations about the produced silica aerogels.

\begin{tabular}{cccc}
\hline Sample ID & $\begin{array}{c}\text { Density } \mathbf{, ~} \mathbf{g} / \mathbf{c m}^{\mathbf{3}} \\
\text { (Eq.1) }\end{array}$ & $\begin{array}{c}\text { \%Porosity } \\
\text { (Eq.2) }\end{array}$ & $\begin{array}{c}\text { \%Volume Shrinkage } \\
\text { (Eq.3) }\end{array}$ \\
\hline SG-IL & $0.45 \pm 0.01$ & $80 \pm 0.012$ & $8.50 \pm 0.010$ \\
\hline SG & $1.23 \pm 0.01$ & $4480 \pm 0.012$ & - \\
\hline
\end{tabular}


Gizli N, Sert Çok S, Koç F. JOTCSA. 2018, 5(2): 663-678.

RESEARCH ARTICLE

It can be stated that the sample prepared without ionic liquid may contain dense regions in the silica network and has large pores. Since ionic liquid controls the solvent evaporation by ensuring elasticity on the pore walls, without ionic liquid as a support, solid skeleton of the silica cluster of sample SG drastically collapse due to the severe capillary tension. Besides, ILembedded sample SG-IL exhibit high porosity with relatively low density. Also, we can say that it remained stable after drying process as the total volumetric shrinkage has a relatively low value of $8.5 \%$.

\section{Visual observations}

\section{Physical appearance}

Figure 3 demonstrates the physical form of two samples SG-IL and SG after ambient pressure drying. As seen from the figure, the physical appearance of silica aerogels dried under ambient conditions strongly affected by the addition of ionic liquid through the synthesis. The sample SG-IL appeared in monolithic form without cracks while the sample SG was grounded into pieces because of capillary forces occurred during the solvent evaporation. On the other hand, the low surface tension and lower vapor pressure of ILs make it possible to sustain the physical stability of silica framework after the subsequent solvent exchange and drying steps.

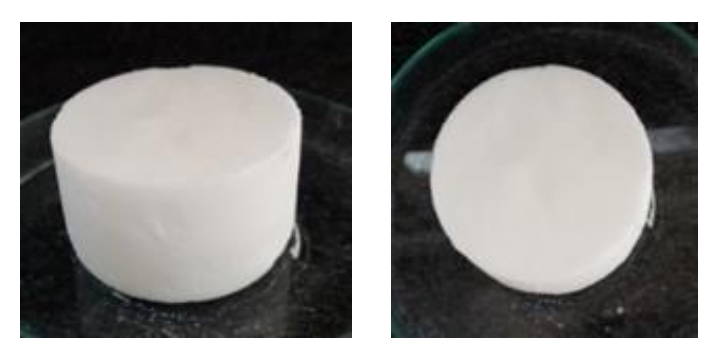

(a)

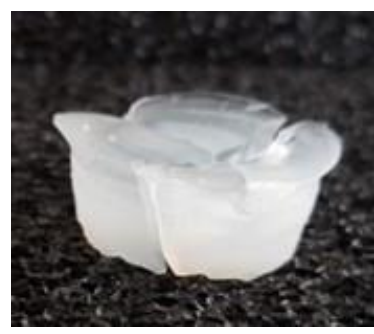

(b)

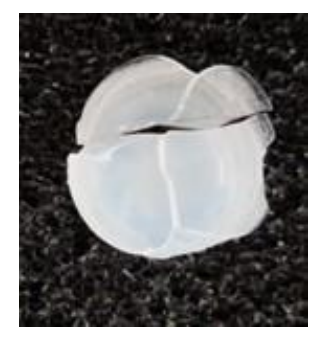

Figure 3. Physical appearances of the samples SG-IL(a) and SG (b).

\section{Gelation time}

Reduction the required time for complete gelation became a major interest in recent studies. An appreciable decrease in a total synthesis time of silica aerogels brings about considerable advantages to these materials for practical purposes. It was suggested in the study of Dourbash et al. that developing one-step sol-gel process instead of time-consuming two-step processes would be preferable (22). In this study, it was observed that amine-rich APTES used as co-precursor eliminated the need for additional base catalyst in the sol-gel method. Regarding its marvelous ability in catalyzing the condensation reactions, APTES significantly reduced to gelation time. Necessary times for complete gelation in this study are observed as $3 \mathrm{~min}$ and $6 \mathrm{~min}$ for the samples of SG-IL and SG, respectively which indicates that ionic liquid supports the catalytic activity of APTES in the condensation reactions, synergistically. 


\section{Morphological properties}

Although the physical appearance of the first sample points out the desired monolithic form under ambient conditions has been achieved, it was not conclusive in visualizing the morphological differences in the porous structures of the samples with or without ionic liquid. The SEM images of the synthesized silica aerogels prepared with/without ionic liquid are demonstrated in Figure 4. Sample SG-IL exhibits a three-dimensional porous network consisting of the spherical silica clusters indicates that typical microstructure pattern of silica aerogels was obtained. Sample SG, however, results in the insufficient porous network. As it is very weak to withstand capillary pressure, it exhibits a rigid structure.
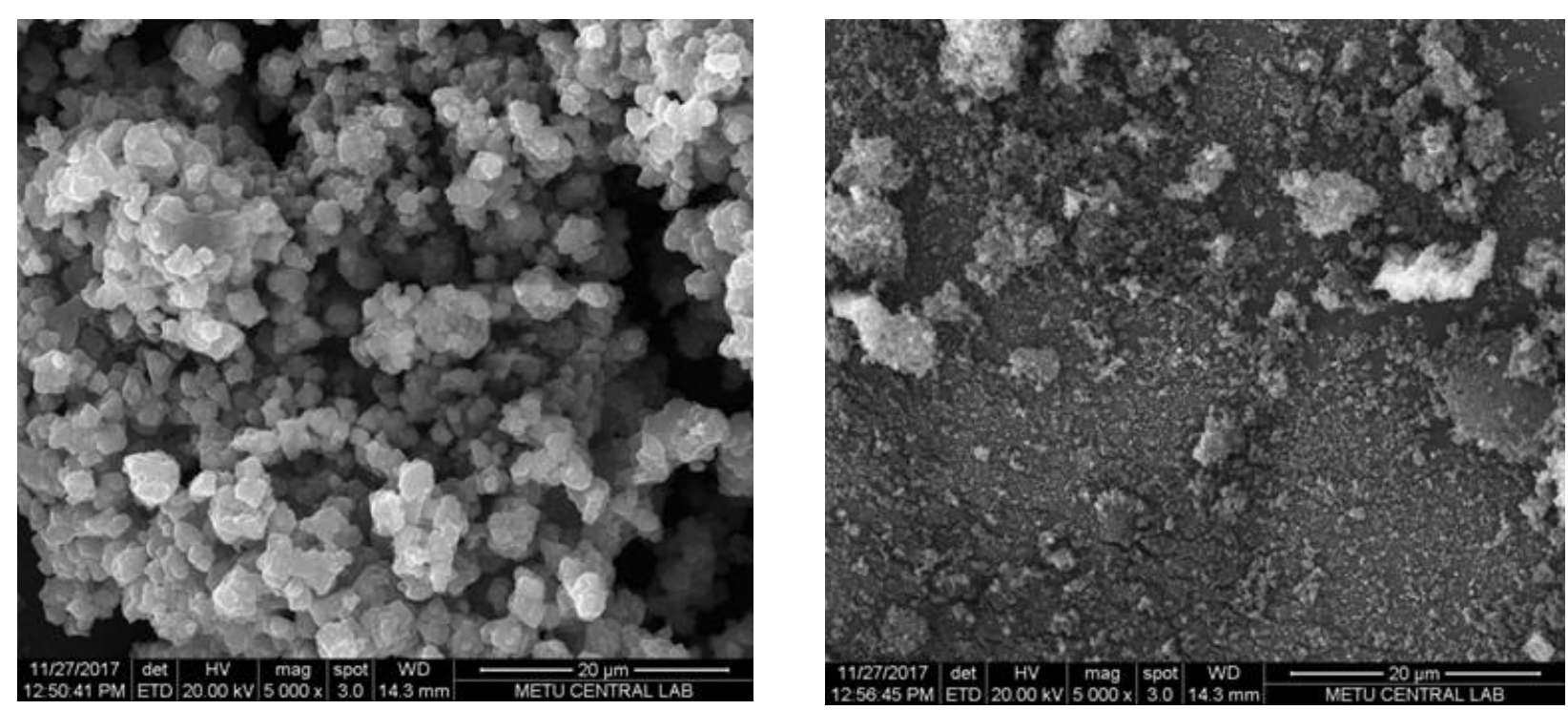

Figure 4. SEM Images of the samples SG-IL and SG with magnification rate is 5000.

\section{FTIR spectra}

FTIR analysis was conducted to reveal related chemical bonding in silica aerogel synthesis and shown in Figure 5. 


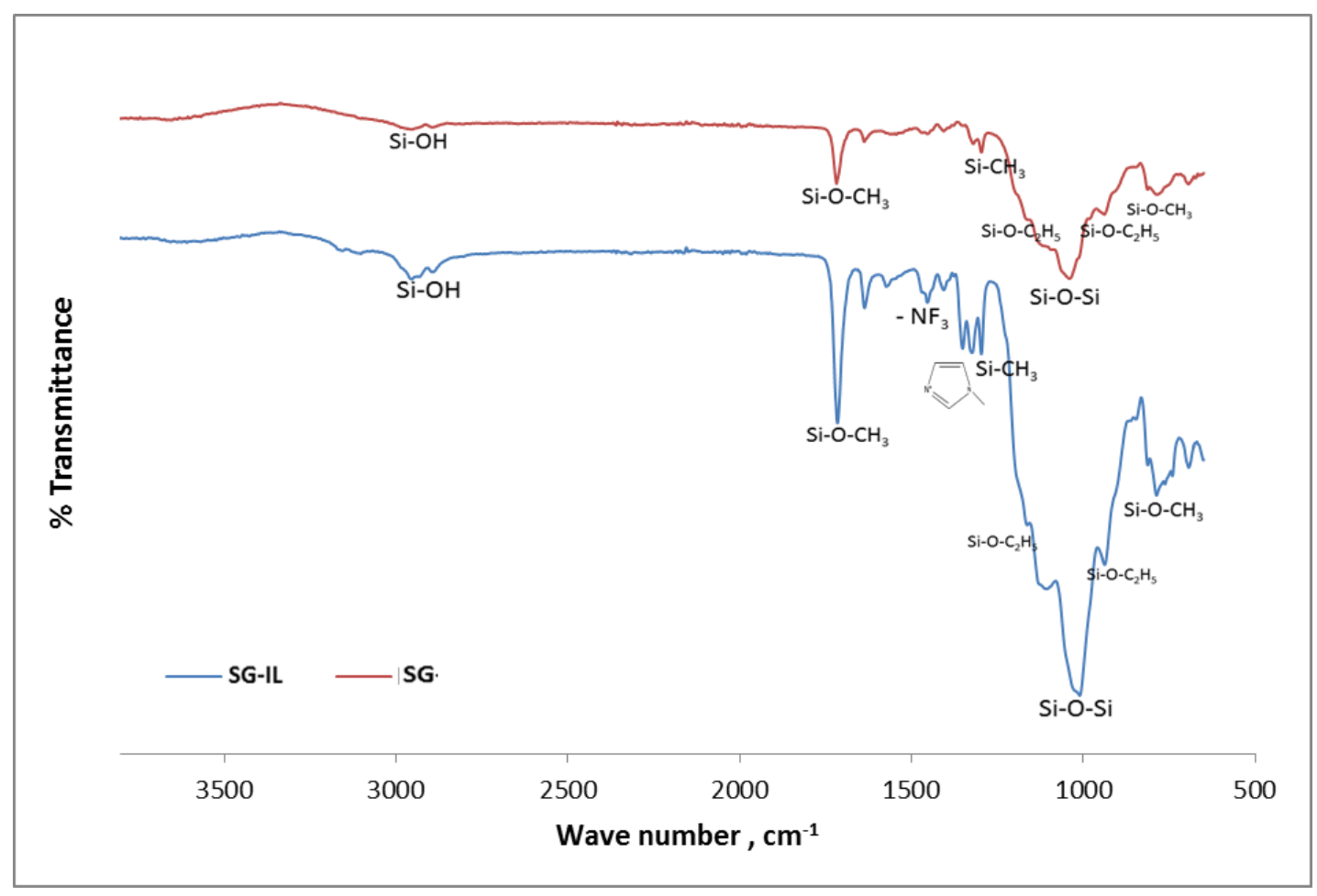

Figure 5. FTIR Spectral results of the samples SG-IL and SG.

The absorption peaks around $1080 \mathrm{~cm}^{-1}$ may be attributed to $\mathrm{Si}-\mathrm{O}-\mathrm{Si}$ bonding confirming the development of the silica network. It is obvious from the spectrum that intensity of the $\mathrm{Si}-\mathrm{O}-\mathrm{Si}$ vibrations increases prominently with the addition of ionic liquid to the sample. Hence, it can be affirmed that ionic liquid is incorporated into the structure of silica aerogels and can improve network formation. The peaks observed the around $1352 \mathrm{~cm}^{-1}$ and $1471 \mathrm{~cm}^{-1}$ may be related to the existence of the imidazolium-based ionic liquid. Distinct $\mathrm{Si}-\mathrm{CH}_{3}$ peaks observed around $785 \mathrm{~cm}^{-1}$ and $1350 \mathrm{~cm}^{-1}$ verify stronger gel network and attachment of the methyl groups to the gel surface may indicate a successful surface modification with MEMO.

\section{TGA/DTG analysis and thermal stability}

Based on the previous characterizations applied through the study, it can be seen that ionic liquids enhanced the formation and conservation of the monolithicity of the samples as well as it served as a catalyst and accelerated the condensation rate in the sol-gel method. TGA analysis was conducted to investigate the effect of the ionic liquid on the thermal stability of the samples. Figure 6 demonstrates the thermal degradation of the Sample SG-IL and Sample SG with respect to temperature. The sample prepared without ionic liquid exhibited a thermal decomposition temperature around $144{ }^{\circ} \mathrm{C}$. It lost almost $12 \%$ of its total weight up to this temperature. Confinement ionic liquid, however, increased the thermal stability of the sample SG-IL by extending the thermal degradation temperature of the sample up to $381^{\circ} \mathrm{C}$. 


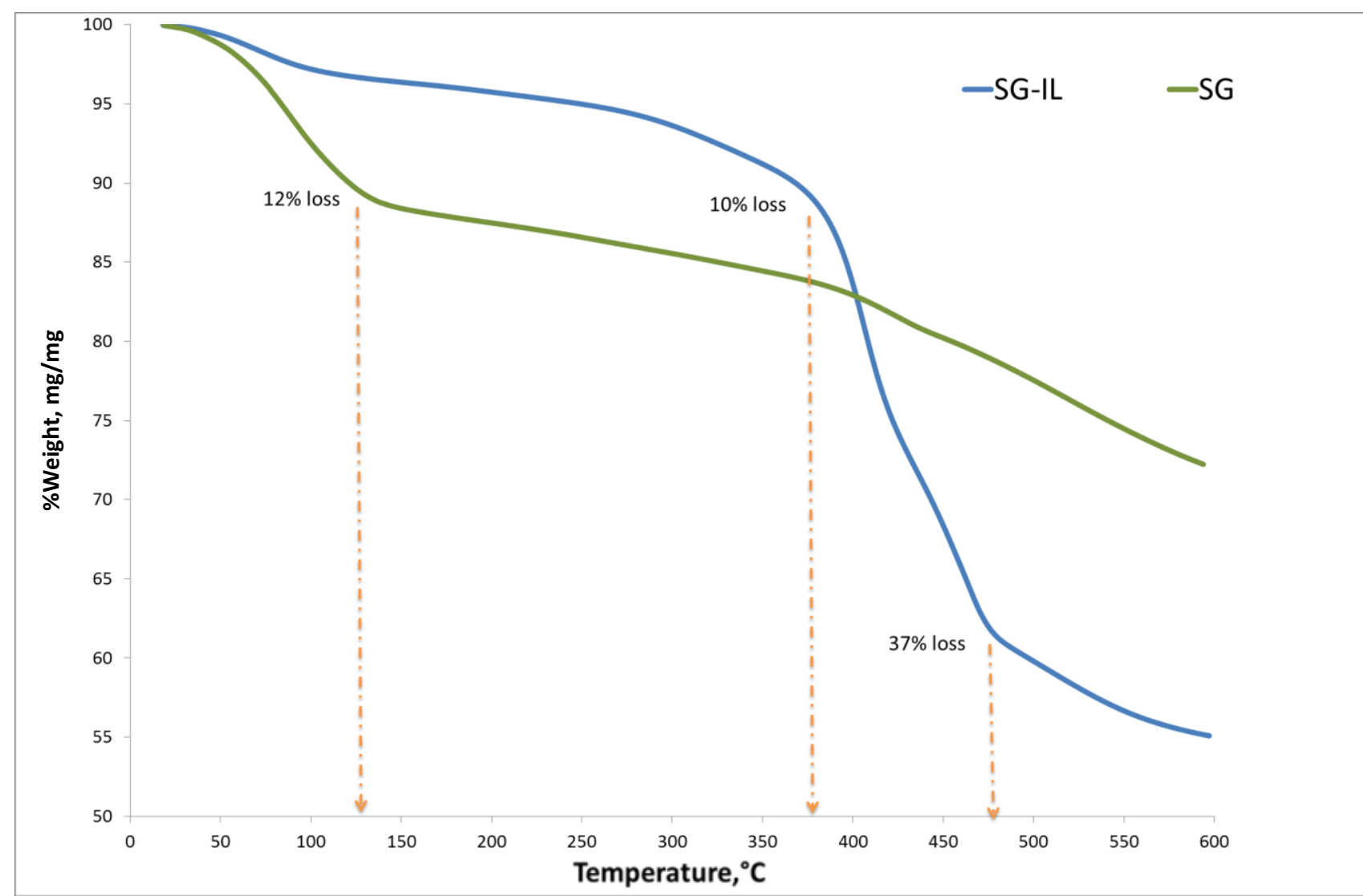

Figure 6. TGA analysis results of the samples SG-IL and SG.

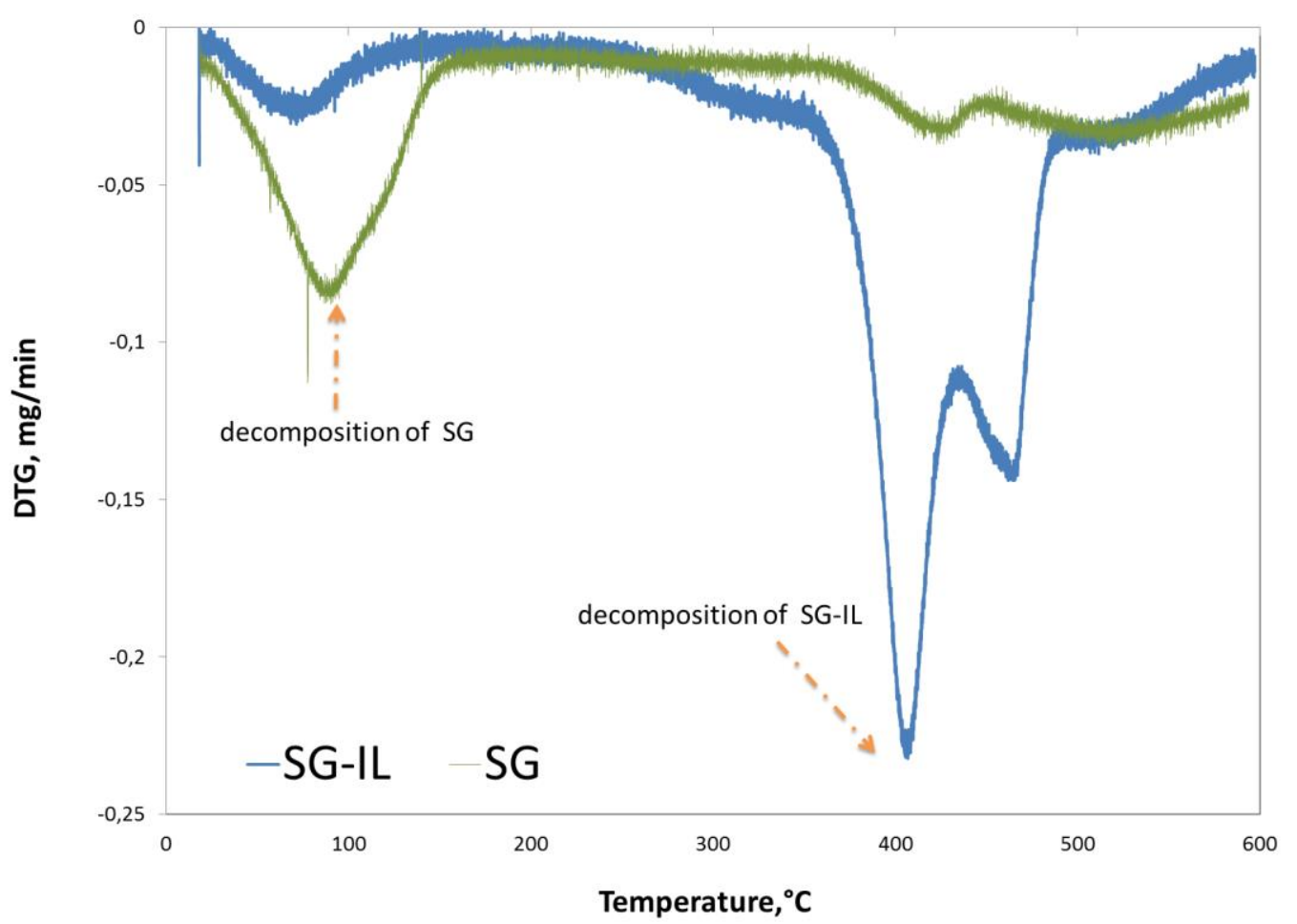

Figure 7. DGT analysis results of the samples SG-IL and SG. 
Gizli N, Sert Çok S, Koç F. JOTCSA. 2018, 5(2): 663-678.

RESEARCH ARTICLE

Then, a drastic change in the total weight of the sample of SG-IL after the temperature of $381^{\circ} \mathrm{C}$ which leads almost two times higher weight loss comparing with the other sample (SG), can be attributed to the degradation of imidazolium based ionic liquid. The results also confirmed with the DTG analysis given in Figure 7. For the ionic liquid embedded samples, having good thermal stability within the temperature range of $0^{\circ} \mathrm{C}-380^{\circ} \mathrm{C}$, can bring the material a valuable characteristic when considering possible target applications such as household thermal insulation material, as catalyst supports or as adsorbents.

\section{Hydrophobicity and contact angle}

The surface modification was performed by replacing hydroxyl groups on the surface of the silica gel with the alkyl groups for the purpose of reducing the surface energy of the gel and turns the sample into hydrophobic form. MEMO was selected as silylating agent and the water re-balancing ability of dried sample SG-IL was determined by measuring the contact angle of water droplet on the sample surface and presented in Figure 8. Then again, surface energy of the sample SG-IL was determined $15.47 \mathrm{mN} / \mathrm{m}$ by the same measurement. For the sample SG, however, contact angle measurement could not be performed because of its fractural structure.

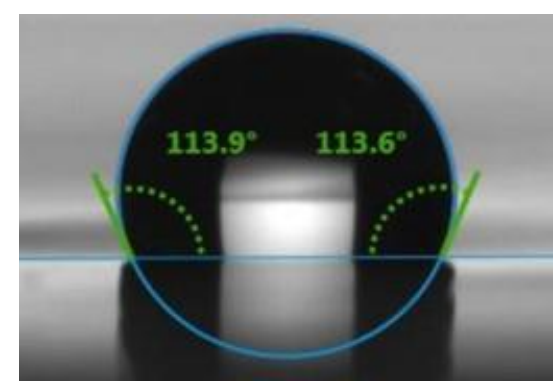

Figure 8. The contact angle value of the sample SG-IL

\section{CONCLUSION}

In this study, monolithic silica gels were synthesized by taking advantage of the synergistic effect of imidazolium-based short-chain ionic liquid and amine-rich co-precursor APTES in the sol-gel method. With the help of these agents, required gelation time was reduced down to 3 min. This collective interaction also eliminated the need for the repeated surface modification periods and hydrophobic characteristic of the samples was achieved by applying one-step surface modification only. Ionic liquid also served as a drying control agent during the solvent evaporation of the gel and contributed in retaining solid skeleton network of silica particles with highly homogeneous pore distribution after ambient pressure drying. It was also observed that thermal stability of these samples highly affected by the presence of ionic liquid.

Due to the obtained superior physical properties such as low density and highly porous structure and high thermal stability, the synthesized samples can be promising candidates for a wide range of applications to be used as thermal and acoustic insulators, as adsorbents 
Gizli N, Sert Çok S, Koç F. JOTCSA. 2018, 5(2): 663-678.

(especially in the $\mathrm{CO}_{2}$ capture applications due to the amine containing functional groups of the samples) as catalyst supports or as energy storage barriers. Synthesizing the samples as monolithic blocks with controlled shape, have also gave them the opportunity to be used more easily for any particular application.

For the further studies, it can be suggested that using ionic liquids along with an amine mediated precursor through the silica aerogel preparation can be an attractive approach.

\section{REFERENCES}

1. Li M, Jiang H, Xu D, Hai O, and Zheng W. Low density and hydrophobic silica aerogels dried under ambient pressure using a new co-precursor method. Journal of Non-Crystalline Solids. 2016 Nov; 452 : 187-193.

2. Li Z, Cheng X, He S, Shi X, Gong L, and Zhang H. "Aramid fibers reinforced silica aerogel composites with low thermal conductivity and improved mechanical performance. Compos. Part A Appl. Sci. Manuf.2016; 84: 316-325.

3. Laskowski J, Milow B, and Ratke L. Aerogel-aerogel composites for normal temperature range thermal insulations. Journal of Non-Crystalline Solids. 2016; 441: 42-48.

4. Soleimani Dorcheh A and Abbasi M. H. Silica aerogel; synthesis, properties and characterization. J. Mater. Process. Technol. 2008; 199-1:10-26.

5. Parmenter K. E. Mechanical properties of silica aerogels. Journal of Non-Crystalline Solids. 1996 Nov; 1: $79-189$

6. Amonette J. E, and Matyáš J. Functionalized silica aerogels for gas-phase purification, sensing, and catalysis: A review. Microporous Mesoporous Materials. 2017; 250: 100-119.

7. Maleki H, Dur L, and Garc C. A. Synthesis and Biomedical Applications of Aerogels: Possibilities and Chal- lenges Hajar. Advance Colloid Interface Science. 2016; 236: 1-27.

8. Buratti C, Merli F, and Moretti E. Aerogel-based materials for building applications: Influence of granule size on thermal and acoustic performance. Energy Buildings. 2017; 152: 472-482.

9. Berthon-Fabry S, Hildenbrand C, Ilbizian P, Jones E, and Tavera S. Evaluation of lightweight and flexible insulating aerogel blankets based on Resorcinol-Formaldehyde-Silica for space applications. Eur. Polym. J. 2017 May; 93: 403-416.

10. Wu C. M, Lin S. Y, and Chen H. L. Structure of a monolithic silica aerogel prepared from a short-chain ionic liquid. Microporous Mesoporous Matererials. 2012; 156: 189-195.

11. Hilonga A, Kim J. K, Sarawade P. B, and Kim H. T. Low-density TEOS-based silica aerogels prepared at ambient pressure using isopropanol as the preparative solvent. J. Alloys Compd.2009; 487: 744750 .

12. Wei T. Y, Chang T. F, Lu S. Y, and Chang Y.C. Preparation of monolithic silica aerogel of low thermal conductivity by ambient pressure drying. J. Am. Ceram. Soc.2007; 90: 2003-2007.

13. Garay Martinez R, Goiti E, Reichenauer G, Zhao S, Koebel M, and Barrio A. Thermal assessment of ambient pressure dried silica aerogel composite boards at laboratory and field scale. Energy Buildings. 2016; 128: 111-118.

14. Mazraeh-Shahi Z. T, Shoushtari A. M, Abdouss M, and Bahramian A. R. Relationship analysis of processing parameters with micro and macro structure of silica aerogel dried at ambient pressure. Journal of Non-Crystalline Solids. 2013; 376: 30-37.

15. Yang $\mathrm{H}$, Kong $\mathrm{X}$, Zhang $\mathrm{Y}, \mathrm{Wu} \mathrm{C}$, and Cao E. Mechanical properties of polymer-modified silica aerogels dried under ambient pressure. Journal of Non-Crystalline Solids. 2011; 357: 3447-3453. 
16. Pons A, Casas L, Estop E, Molins E, Harris K. D. M, and Xu M. A new route to aerogels: Monolithic silica cryogels. Journal of Non-Crystalline Solids. 2012; 358: 461-469.

17. Ivanova M, Kareth S, Spielberg E. T, Mudring A. V, and Petermann M. Silica ionogels synthesized with imidazolium based ionic liquids in presence of supercritical CO2. J. Supercrit. Fluids. 2014; 105: 6065.

18. Zhang J, Ma Y, Shi F, Liu L, and Deng Y. Microporous and Mesoporous Materials Room temperature ionic liquids as templates in the synthesis of mesoporous silica via a sol - gel method. 2009; 119: 97103.

19. Ivanova M, Kareth S, and Petermann M. Supercritical carbon dioxide and imidazolium based ionic liquids applied during the sol-gel process as suitable candidates for the replacement of classical organic solvents. J. Supercritical Fluids. 2017 July; 132: 76-82.

20. Karout A and Pierre A. C. Silica xerogels and aerogels synthesized with ionic liquids. 2007; 353: 2900-2909.

21. Karout A and Pierre A. C. Silica gelation catalysis by ionic liquids. Catal. Commun. 2009; 10-4: 359361.

22. Dourbash A, Motahari S, and Omranpour H. Effect of water content on properties of one-step catalyzed silica aerogels via ambient pressure drying. Journal of Non-Crystalline Solids. 2014; 405: 135-140.

23. Li S, Ren H, Zhu J, Bi Y, Xu Y, and Zhang L. Facile fabrication of superhydrophobic, mechanically strong multifunctional silica-based aerogels at benign temperature. Journal of Non-Crystalline Solids. 2017; 473: 59-63.

24. Yu H, Liang X, Wang J, Wang $M$, and Yang S. Preparation and characterization of hydrophobic silica aerogel sphere products by co-precursor method. Solid State Sci. 2015; 48: 155-162. 
